ARTIGO ORIGINAL ORIGINAL ARTICLE

Palavras-chave:

câncer de próstata, metástase, custos, saúde suplementar

\section{Câncer de próstata com metástase óssea: impacto econômico para o Sistema de Saúde Suplementar brasileiro}

\author{
Prostate cancer with bone metastasis: budget impact \\ to the Brazilian Private Heathcare System \\ Enzo Waiti Asano', Hellen Soo Jin Kim², Mariana Carvalho del \\ Aguila², Sandra Navarro ${ }^{2}$, Roberta Arinelli Fernandes ${ }^{1}$ \\ DOI: 10.21115/JBES.v10.n2.p157-164
}

\begin{abstract}
RESUMO
Objetivo: $O$ objetivo deste trabalho foi avaliar o impacto de custo total do tratamento de câncer de próstata com metástase óssea na perspectiva do Sistema de Saúde Suplementar (SSS) brasileiro. Métodos: Trata-se de um estudo retrospectivo de coorte conduzido de um banco de dados administrativos fornecidos pela empresa Orizon. A população consistiu em pacientes com registro de câncer de próstata com metástases ósseas, ou procedimentos relacionados, que foram registrados em entradas hospitalares ou ambulatoriais e receberam pelo menos um tipo de tratamento. A perspectiva da análise foi o SSS e o horizonte temporal, uma mediana de 181 dias. O desfecho primário foi o custo global, no qual foram contemplados gastos com exames, procedimentos, materiais e medicamentos. A partir do desfecho primário, foram calculados o custo por paciente, o número de atendimentos por paciente e o custo por atendimento. Adicionalmente foram analisados os custos relacionados aos diferentes subgrupos de tratamentos destinados à doença. Resultados: $\bigcirc$ custo global relacionado aos atendimentos foi de $\mathrm{R} \$ 21.391 .034(\mathrm{n}=181)$, o custo médio resultou em $\mathrm{R} \$$ 118.183 por paciente, com média de 10,48 atendimentos por paciente e R\$11.282 por atendimento. Pela análise isolada dos cenários, os custos nos ambientes hospitalar (R\$11.452.496) e ambulatorial ( $R \$$ 9.938.537) demonstraram que houve mais gastos relacionados aos procedimentos hospitalares. Conclusões: $\mathrm{O}$ estudo demonstrou elevado impacto econômico de tratamento de câncer de próstata com metástases ósseas no SSS, devido principalmente a maior necessidade de quantidade de hospitalizações com mais tempo de permanência.
\end{abstract}

\section{Keywords:}

prostate cancer, metastasis, costs, supplementary healthcare

\begin{abstract}
Objective: The objective of this study was to evaluate the total cost impact of treatment of prostate cancer with bone metastasis under the Brazilian Private Healthcare System (SSS) perspective. Methods: This is a retrospective cohort study conducted from an administrative database provided by Orizon. The population consisted in patients with prostate cancer with bone metastasis registry, or related procedures, who were registered at hospital or ambulatory admission, and received at least one type of treatment. The perspective of the analysis was SSS and the time horizon was a median of 181 days. The primary outcome was global cost, considering expenses with exams, procedures, materials and drugs. From the primary outcome, cost per patient, number of visits per patient, and visit cost were calculated. Additionally, the costs related to the different subgroups of disease treatments
\end{abstract}


were analyzed. Results: The global cost related to visits was $R \$ 21,391,034(n=181)$, the average cost resulted in $R \$ 118,183$ per patient, with an average of 10.48 visits per patient and $R \$ 11,282$ per visit. By the isolated analysis of the scenarios, the costs in the hospital $(R \$ 11,452,496)$ and ambulatory $(R \$$ 9,938,537) environment demonstrated more expenses related to hospital procedures. Conclusions: The study demonstrated elevated budget impact of prostate cancer with bone metastasis treatment on the SSS, mainly due to greater need for the number of hospitalizations with a longer stay.

\section{Introdução}

O câncer de próstata é considerado o segundo tipo de câncer mais comum e a quinta causa de morte por neoplasias na população masculina no mundo (World Health Organization, 2012). Pode seguir com uma evolução silenciosa e assintomática ou apresentar inicialmente apenas sintomas semelhantes aos do crescimento benigno da próstata, como dificuldade de urinar e necessidade de urinar mais vezes durante o dia (Ministério da Saúde - Brasil, 2016a).

No Brasil, de acordo com o Instituto Nacional do Câncer, foram estimados 68.220 novos casos em 2018, com incidência anual de 66,12 por 100 mil homens (Ministério da Saúde, 2018). Dessa forma, quando não considerados os tumores de pele não melanoma, o câncer de próstata é o mais incidente entre os homens em todas as regiões do país, com taxas de $98,85,69,83,66,75,56,17$ e 29,41 por 100 mil homens no Sul, Sudeste, Centro-Oeste, Nordeste e Norte, respectivamente (Ministério da Saúde, 2018). Sua taxa anual de mortalidade é de 16 por 100 mil habitantes e, a cada ano, cerca de 14.500 óbitos relacionados ao câncer de próstata são registrados no Brasil (Jerez-Roig et al., 2014). Entre os pacientes que evoluem a óbito por câncer, $90 \%$ apresentam evidências de metástase óssea (Bubendorf et al., 2000).

Nos Estados Unidos, 12\% dos pacientes apresentam doença localmente avançada e 4\%, doença metastática no momento do diagnóstico (National Cancer Institute). Porém, no Brasil, estima-se que 30\% dos pacientes já apresentem doença metastática ao diagnóstico (Agência Brasil, 2017). Apesar de a doença localizada ao diagnóstico ser passível de tratamento com finalidade curativa, cerca de 30\% dos pacientes apresentam recorrência da doença, com possibilidade de progressão para a forma metastática do câncer de próstata e para o comportamento de resistência à castração (National Cancer Institute; Chi et al., 2009).

O esqueleto é o local mais comum de metástases em pacientes com câncer de próstata (Gandaglia et al., 2014). A fisiopatologia da formação de metástase óssea ainda não foi totalmente elucidada, mas modelos experimentais e evidências clínicas têm auxiliado na evolução desse conhecimento (Manca et al., 2017). As metástases ósseas são uma causa clinicamente significativa de morbidade e mortalidade, muitas vezes resultando em dor óssea, fratura patológica ou compressão medular, que requerem tratamento, muitas vezes cirúrgico (Shore, 2015). Os eventos esqueléticos também costumam cursar com incapacidade funcional e queda na qualidade de vida relacionada à saúde (Shore, 2015).

Em razão da alta incidência mundial, o câncer de próstata apresenta elevada carga econômica para os sistemas de saúde.

O conhecimento dos custos associados a cada etapa evolutiva de uma patologia é capaz de auxiliar no processo de gestão eficiente dos sistemas de saúde. Este estudo teve o objetivo de descrever a utilização de recursos e os custos médicos associados ao tratamento do câncer de próstata com metástase óssea, sob a perspectiva do Sistema de Saúde Suplementar, no Brasil.

\section{Métodos}

Um estudo retrospectivo de coorte foi conduzido com uma base de dados administrativa fornecida pela empresa Orizon, responsável pela consolidação de informações obtidas por diferentes tipos de operadoras privadas de saúde no Brasil, totalizando a administração de cerca de 18 milhões de vidas na Saúde Suplementar Brasileira, ou 35\% do mercado. A análise abrangeu o período de janeiro de 2010 a dezembro de 2015.

Para entrada na coorte, foram considerados os seguintes critérios de inclusão:

- Pacientes com diagnóstico de câncer de próstata, registrados na base de dados durante o período de janeiro de 2010 a dezembro de 2013, de acordo com a Classificação Internacional de Doenças e Problemas Relacionados à Saúde, 10a edição (CID-10), como C61.

- Registro de metástases ósseas (CID C795 e M97) ou procedimentos relacionados, após registro de câncer de próstata, durante 0 período de janeiro de 2010 a abril de 2015.

- Pacientes que receberam pelo menos um tratamento relacionado ao câncer de próstata (terapia hormonal $[T H]$, radioterapia [RT], terapia oncológica [TO], definida como qualquer tipo de antineoplásico [citotóxico ou agentes de terapia-alvo] ou medicamentos paliativos para dor óssea) e cirurgia (SG) ou foram submetidos a um procedimento ósseo (PO), após registro de câncer de próstata, durante o período de análise (janeiro de 2010 a dezembro de 2015).

- Pacientes que tiveram pelo menos um registro de entrada hospitalar ou ambulatorial, após registro 
de câncer de próstata e durante o período de análise (janeiro de 2010 a dezembro de 2015).

A primeira extração de informações do banco de dados que reuniu pacientes com diagnóstico de câncer de próstata teve início em janeiro de 2010 e encerrou em dezembro de 2013, considerado o período de seleção. Os demais critérios de inclusão foram avaliados no período de janeiro de 2010 a abril de 2015. O acompanhamento dos pacientes teve início após a primeira admissão hospitalar e foi mantido até a data final do estudo, em dezembro de 2015. A Figura 1 apresenta a sequência temporal para a seleção de pacientes e análise de dados.

A análise teve como objetivo primário a avaliação dos custos associados ao tratamento do câncer de próstata com metástase óssea, sendo os custos classificados como exames, procedimentos, materiais, medicamentos, gases medicinais, pacotes e outras taxas.

Para todos os atendimentos e para os subgrupos de atendimentos hospitalar e ambulatorial, foram considerados os desfechos:

- Custo global, segmentado em exames, procedimentos, materiais, medicamentos, gases medicinais, pacotes e outras taxas, custo por paciente e custo por atendimento.

- Custo por paciente, calculado pela divisão do custo global pela coorte total de pacientes.

- Número de atendimentos por paciente, calculado com a soma do número de atendimentos dividido pela coorte total de pacientes, durante o período de acompanhamento.

- Custo por atendimento, calculado pela divisão do custo por paciente pelo número de atendimentos por paciente.

Exclusivamente para os atendimentos hospitalares, foram avaliados o tempo médio de duração do atendimento, calculado como a soma das durações dos atendimentos hospita-

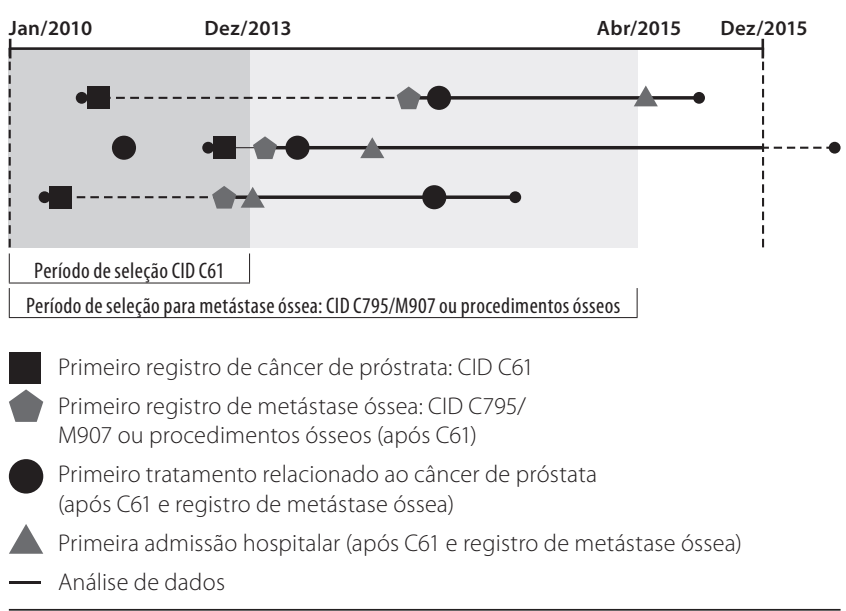

Figura 1. Sequência temporal de seleção de pacientes e análise de dados. lares dividida pela coorte total de pacientes, e o custo por dia de atendimento, calculado pela divisão do custo por atendimento pelo número médio da duração do atendimento.

Diferentemente das análises anteriores, para os desfechos secundários, foi considerada a coorte de cada subgrupo por tipo de tratamento: SG, RT, TO, TH e PO. Adicionalmente, avaliou-se o intervalo médio entre cada atendimento.

A análise de subgrupos de tratamento foi realizada após validação de um especialista médico e focada em registros exclusivamente relacionados ao tipo de tratamento de interesse. Registros de pacientes que receberam mais de um tipo de tratamento, para os quais não foi possível distinguir os custos e recursos específicos de cada modalidade, foram excluídos da análise de subgrupos.

A análise descritiva estatística foi realizada por meio de uma plataforma de software MicroStrategy version 10.1 (MicroStrategy Inc., Virginia, EUA). Uma vez que essa análise retrospectiva foi baseada em revisão de banco de dados, cujas informações são agregadas, sem possibilidade de identificação individual dos sujeitos, não houve necessidade de avaliação por Comitê de Ética em Pesquisa nem tampouco aplicação de termo de consentimento livre e esclarecido, de acordo com a resolução no 510, de 7 de abril de 2016 (Ministério da Saúde - Brasil, 2016b).

Com o objetivo de projetar o impacto para a realidade atual, os resultados de custos foram ajustados de acordo com os índices de inflação de 2016 (6,29\%) e 2017 (2,95\%), segundo o Índice Nacional de Preços ao Consumidor Amplo (IPCA) (Instituto Brasileiro de Geografia e Estatística - IBGE, 2017).

\section{Resultados}

\section{Coorte de pacientes}

No período inicial de seleção, foram identificados 35.265 pacientes com CID específico para câncer de próstata registrados no banco de dados, dos quais 181 se encaixavam nos critérios de inclusão do estudo. O fluxo de seleção de pacientes está apresentado na Figura 2. A mediana de tempo de acompanhamento foi de 181 dias.

\section{Distribuição de tratamentos}

Durante o período de acompanhamento, a distribuição de tratamentos entre os pacientes demonstrou que:

- 21,5\% (39/181) foram submetidos a apenas um tipo de tratamento.

- 37,5\% (68/181) receberam três tipos de tratamento (TH, RT e TO).

- 7,7\% (14/181) pacientes foram submetidos exclusivamente a procedimentos para metástases ósseas, e não foram incluídos na distribuição de tratamento. A Figura 3 mostra a distribuição de pacientes pelos tipos de tratamentos. 


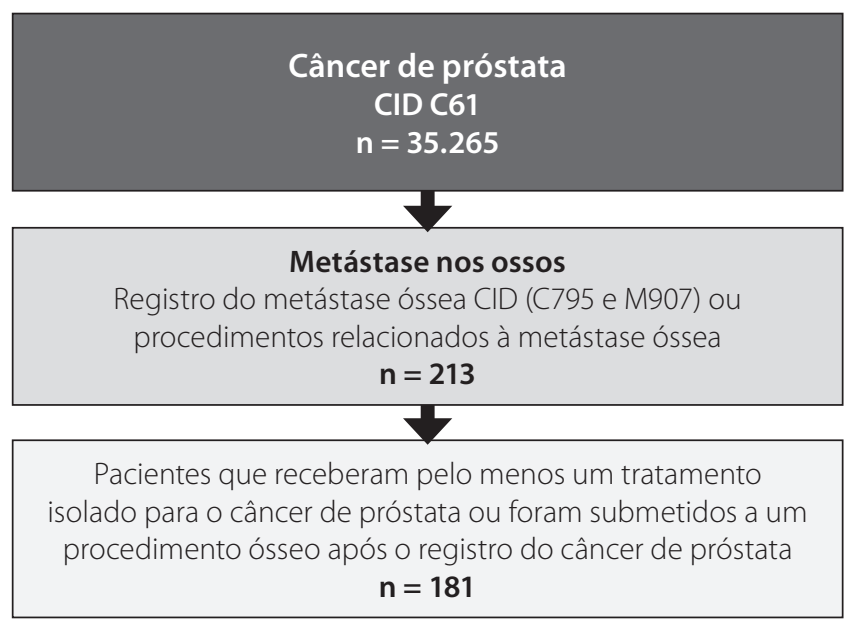

Figura 2. Fluxograma de seleção de pacientes.

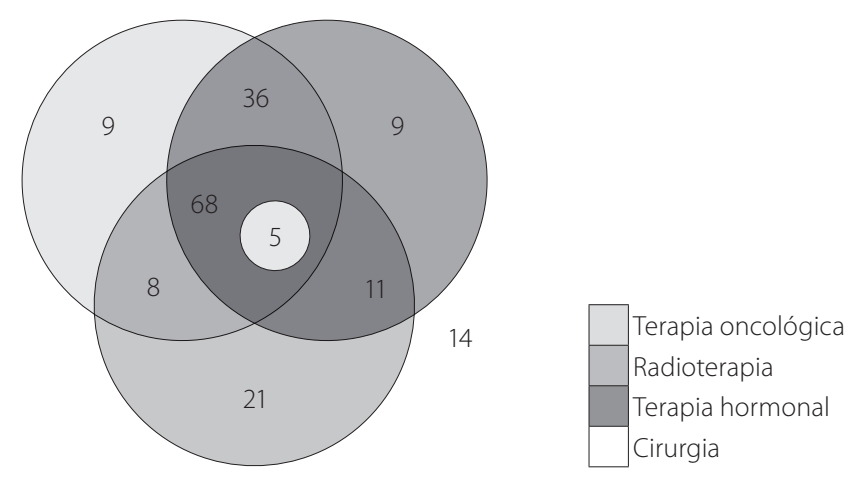

\begin{tabular}{lc}
\hline Terapia oncológica (tratamento isolado) & $\mathbf{9}$ \\
\hline Radioterapia (tratamento oncológico) & $\mathbf{2 1}$ \\
\hline Terapia hormonal (tratamento isolado) & $\mathbf{9}$ \\
\hline Cirurgia (isolada) & $\mathbf{0}$ \\
\hline Terapia oncológica + Radioterapia & $\mathbf{8}$ \\
\hline Terapia oncológica + Terapia hormonal & $\mathbf{3 6}$ \\
\hline Terapia oncológica + Cirurgia & $\mathbf{0}$ \\
\hline Radioterapia + Terapia hormonal & $\mathbf{1 1}$ \\
\hline Radioterapia + Cirurgia & $\mathbf{0}$ \\
\hline Terapia hormonal + Cirurgia & $\mathbf{0}$ \\
\hline Terapia oncológica + Radioterapia + Terapia hormonal & $\mathbf{6 8}$ \\
\hline Terapia oncológica + Terapia hormonal + Cirurgia & $\mathbf{0}$ \\
\hline Terapia oncológica + Radioterapia + Cirurgia & $\mathbf{0}$ \\
\hline Radioterapia + Terapia hormonal + Cirurgia & $\mathbf{0}$ \\
\hline Terapia oncológica + Radioterapia + Terapia hormonal + Cirurgia & $\mathbf{5}$ \\
\hline Nenhum & $\mathbf{1 4}$ \\
\hline Total & $\mathbf{1 8 1}$ \\
\hline
\end{tabular}

Figura 3. Distribuição de pacientes por tipos de tratamento.

\section{Custos e atendimentos}

Somando os ambientes ambulatorial e hospitalar, o resultado do custo global relacionado aos atendimentos para a coorte atingiu R\$21.391.034,29. Medicamentos foram responsáveis pela maior parte dos custos ( $R \$ 12.160 .647,90 ; 56,85 \%)$, seguidos por materiais (R\$3.952.465,78; 18,48\%), taxas diversas (R\$ 2.299.293,22; 10,75\%), procedimentos ( $R \$ 1.546 .887,44$;
7,23\%), exames (R\$ 903.640,13; 4,22\%), gases medicinais ( $R \$$ 352.204,29; 1,65\%) e pacotes (R\$ 175.895,54; 0,82\%) (Tabela 1).

Ao avaliar os atendimentos ambulatoriais isoladamente, o custo ambulatorial para a coorte atingiu o valor de R\$ 9.938.537,52. Nesse ambiente, os medicamentos foram responsáveis pela maior parte dos custos (87,77\%) e cada uma das demais categorias de custos representa menos de $5 \%$ da composição do custo ambulatorial.

O custo dos atendimentos hospitalares totalizou R\$ 11.452.496,78 para a coorte. Nesse ambiente, as categorias de custo tiveram uma distribuição diferente da observada nos atendimentos ambulatoriais. As demais categorias são as principais responsáveis pelos custos (65,69\%), sendo 30,46\% gastos com materiais, 19,04\% com taxas diversas, 9,3\% com procedimentos e $6,88 \%$ com exames, e os custos com medicamentos caíram significativamente, representando 30,02\%.

A Figura 4 apresenta a composição do custo global segmentada por tipo de atendimento e categoria de custo.

Considerando que cada paciente requer uma média de 8,91 atendimentos ambulatoriais e 1,56 atendimento hospitalar, aplicando-se um custo por atendimento de R\$ 6.161,52 e $R \$ 40.468,19$, a análise resultou em um custo médio de R\$ $54.909,05$ e R\$ 63.273,46 por paciente para os ambientes ambulatorial e hospitalar, respectivamente.

Além disso, para os atendimentos hospitalares, foram constatados tempo médio por atendimento de 10,63 dias e custo por dia de atendimento de $\mathrm{R} \$ 3.808,61$.

Considerando o tempo de acompanhamento da coorte de 181 pacientes (mediana: 181 dias), a análise resultou em um custo por paciente de $\mathrm{R} \$ 118.183$, com média de 10,48 atendimentos por paciente, resultando em um custo por atendimento de $\mathrm{R} \$ 11.282,19$ (Tabela 2).

\section{Custos e atendimentos por tratamento}

Dos custos ambulatoriais, foi possível distinguir os custos dos registros com utilização isolada dos tratamentos TH, RT, TO e PO. Os registros de TO foram responsáveis pela maior parte dos custos (R\$ 1.642.564,65; 16,53\%), seguidos por TH (R\$ 1.290.951,52; 12,99\%), RT (R\$ 422.789,39; 4,25\%) e PO (R\$ $16.407,53 ; 0,17 \%$ ). A soma desses tratamentos totalizou 33,94\% (R\$3.372.713,08) dos custos ambulatoriais da coorte, sendo o restante dos custos ambulatoriais $(66,06 \%)$ oriundos dos registros nos quais não foi possível distinguir os custos e recursos específicos de cada modalidade.

Os tratamentos TH e TO apresentaram um perfil de custos semelhante, sendo o custo com medicamentos responsável por $94,8 \%$ e $88,09 \%$ do custo ambulatorial, respectivamente. Por outro lado, RT teve $91,19 \%$ de seus custos relacionados a procedimentos. PO apresentou $72,94 \%$ e $20,57 \%$ de seus custos associados a procedimentos e materiais, respectivamente (Tabela 3). 
Tabela 1. Composição de custo da coorte de pacientes por categoria

\begin{tabular}{lccc}
\hline Categoria de custo & Ambulatorial $^{+}(\mathbf{n}=\mathbf{1 8 1})$ & Hospitalar $^{+}(\mathbf{n}=\mathbf{1 8 1})$ & Custo global $(\mathbf{n}=\mathbf{1 8 1})$ \\
\hline Medicamentos & $\mathrm{R} \$ 8.723 .103,02(87,77 \%)$ & $\mathrm{R} \$ 3.437 .544,88(30,02 \%)$ & $\mathrm{R} \$ 12.160 .647,90(56,85 \%)$ \\
\hline Materiais & $\mathrm{R} \$ \mathbf{4 6 4 . 5 9 6 , 6 5 ( 4 , 6 7 \% )}$ & $\mathrm{R} \$ 3.487 .869,13(30,46 \%)$ & $\mathrm{R} \$ 3.952 .465,78(18,48 \%)$ \\
\hline Taxas diversas & $\mathrm{R} \$ 118.786,29(1,20 \%)$ & $\mathrm{R} \$ 2.180 .506,93(19,04 \%)$ & $\mathrm{R} \$ 2.299 .293,22(10,75 \%)$ \\
\hline Procedimentos & $\mathrm{R} \$ 480.353,52(4,83 \%)$ & $\mathrm{R} \$ 1.066 .533,91(9,31 \%)$ & $\mathrm{R} \$ 1.546 .887,44(7,23 \%)$ \\
\hline Exames & $\mathrm{R} \$ 115.424,62(1,16 \%)$ & $\mathrm{R} \$ 788.215,51(6,88 \%)$ & $\mathrm{R} \$ 903.640,13(4,22 \%)$ \\
\hline Gases medicinais & $\mathrm{R} \$ 142,53(0,00 \%)$ & $\mathrm{R} \$ 352.061,75(3,07 \%)$ & $\mathrm{R} \$ 352.204,29(1,65 \%)$ \\
\hline Pacotes & $\mathrm{R} \$ 36.130,88(0,36 \%)$ & $\mathrm{R} \$ 139.764,66(1,22 \%)$ & $\mathrm{R} \$ 175.895,54(0,82 \%)$ \\
\hline Total & $\mathrm{R} \$ 9.938 .537,52(100 \%)$ & $\mathrm{R} \$ 11.452 .496,78(100 \%)$ & $\mathrm{R} \$ 21.391 .034,29(100 \%)$ \\
\hline
\end{tabular}

† Percentuais calculados com base no total de cada ambiente.

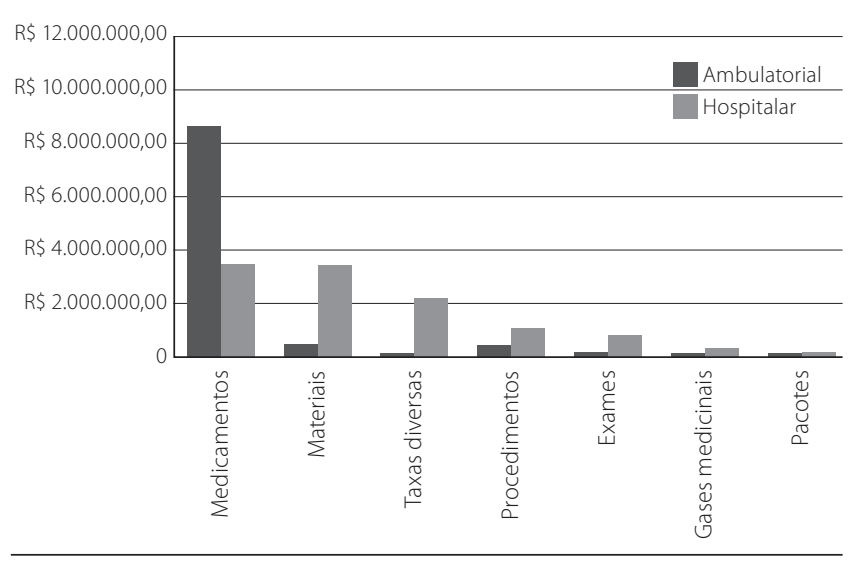

Figura 4. Composição de custo.
Da análise por tipo de tratamento do ambiente ambulatorial, os custos por paciente da TH e da TO se destacam, R\$ 17.445,29 e R\$17.110,05, respectivamente (Tabela 4).

Dos quatro tratamentos avaliados, a TO apresentou maior custo total, porém, devido ao maior volume de atendimentos registrados, apresentou custo por atendimento menor que o da TH e da RT.

Dos custos hospitalares, foram encontrados registros da utilização isolada dos tratamentos TH, RT, TO, SG e PO. Os registros de TH foram responsáveis pela maior parte dos custos ( $R \$ 5.671 .637,56 ; 49,52 \%)$, seguidos por TO (R\$ 1.292.155,30; 11,28\%), PO (R\$ 863.366,19; 7,54\%), RT (R\$ 76.388,93; 0,67\%) e SG (R\$23.106,46; 0,02\%). A soma desses tratamentos totalizou

Tabela 2. Custo médio por paciente, número de atendimentos por paciente, custo por atendimento

\begin{tabular}{lccc}
\hline Desfecho & Ambulatorial $(\mathbf{n}=\mathbf{1 8 1})$ & Hospitalar $(\mathbf{n = 1 8 1 )}$ & Coorte $(\mathbf{n}=\mathbf{1 8 1})$ \\
\hline Custo por paciente & $\mathrm{R} \$ 54.909,05$ & $\mathrm{R} \$ 63.273,46$ & $\mathrm{R} \$ 118.183$ \\
\hline Número de atendimentos & 1.613 & 283 & 1.896 \\
\hline Atendimentos por paciente & 8,91 & 1,56 & 10,48 \\
\hline Custo por atendimento & $\mathrm{R} \$ 6.161,52$ & $\mathrm{R} \$ 40.468,19$ & $\mathrm{R} \$ 11.282,19$ \\
\hline $\begin{array}{l}\text { Tempo médio por } \\
\text { atendimento (dias) }\end{array}$ & - & 10,63 & - \\
\hline Custo por dia de atendimento & - & $\mathrm{R} \$ 3.808,61$ & - \\
\hline
\end{tabular}

Tabela 3. Custo ambulatorial por tipo de tratamento

\begin{tabular}{|c|c|c|c|c|}
\hline Tipo de custo & TH $(n=74)$ & $\operatorname{RT}(n=101)$ & TO $(n=96)$ & $\mathrm{PO}(\mathrm{n}=6)$ \\
\hline Medicamentos & $\mathrm{R} \$ 1.223 .826,87(94,80 \%)$ & $R \$ 3.260,29(0,77 \%)$ & $\mathrm{R} \$ 1.446 .958,17$ (88,09\%) & $R \$ 186,08(1,13 \%)$ \\
\hline Materiais & $\mathrm{R} \$ 17.475,96(1,35 \%)$ & $\mathrm{R} \$ 3.992,00(0,94 \%)$ & $\mathrm{R} \$ 108.527,01(6,61 \%)$ & $R \$ 3.375,26(20,57 \%)$ \\
\hline Taxas diversas & $\mathrm{R} \$ 14.371,41(1,11 \%)$ & $\mathrm{R} \$ 3.910,93(0,93 \%)$ & $\mathrm{R} \$ 29.971,60(1,82 \%)$ & $R \$ 711,23(4,33 \%)$ \\
\hline Procedimentos & $\mathrm{R} \$ 9.114,64(0,71 \%)$ & $\mathrm{R} \$ 385.545,91$ (91,19\%) & $\mathrm{R} \$ 21.410,34(1,30 \%)$ & $\mathrm{R} \$ 11.966,89(72,94 \%)$ \\
\hline Exames & $\mathrm{R} \$ 24.305,12(1,88 \%)$ & $\mathrm{R} \$ 6.037,78(1,43 \%)$ & $\mathrm{R} \$ 34.027,91(2,07 \%)$ & $R \$ 168,07(1,02 \%)$ \\
\hline Gases medicinais & - & $\mathrm{R} \$ 4,66(0,00 \%)$ & $\mathrm{R} \$ 22,98(0,00 \%)$ & - \\
\hline Pacotes & $\mathrm{R} \$ 1.857,53(0,14 \%)$ & $R \$ 20.037,82(4,74 \%)$ & $\mathrm{R} \$ 1.646,64(0,10 \%)$ & - \\
\hline Total & $\mathrm{R} \$ 1.290 .951,52(100,00 \%)$ & $\mathrm{R} \$ 422.789,39(100,00 \%)$ & $\mathrm{R} \$ 1.642 .564,65(100,00 \%)$ & $\mathrm{R} \$ 16.407,53(100,00 \%)$ \\
\hline
\end{tabular}

TH: terapia hormonal; RT: radioterapia; TO: terapia oncológica; PO: procedimento ósseo. 
69,21\% (R\$ 7.926.654,44) dos custos hospitalares da coorte (Tabela 5).

Apenas um paciente realizou SG isoladamente, uma única vez, com custo de $\mathrm{R} \$ 23.106,46$ durante 19 dias de atendimento.

Na avaliação do custo hospitalar por tratamento, verificou-se diminuição da representatividade dos medicamentos na composição de custo da TH, 94,80\% versus 26,54\%, para os ambientes ambulatorial e hospitalar, respectivamente. Em menor proporção, esse mesmo fato ocorreu para a TO, $88,09 \%$ versus 50,74\%, para os ambientes ambulatorial e hospitalar, respectivamente.

TO e TH apresentaram custos por pacientes elevados,

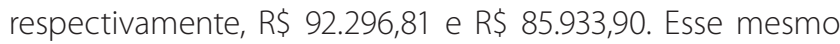
comportamento foi observado para o custo por atendimento desses dois tratamentos. Porém, para TO e TH, o custo por dia de tratamento caiu drasticamente devido à maior permanência do paciente nesses atendimentos, respectivamente, 11,86 e 10,71 dias (Tabela 6).

\section{Discussão}

O custo global relacionado aos atendimentos para a coorte ultrapassou $\mathrm{R} \$ 21$ milhões. A análise resultou em um custo médio de R\$ 118.183 por paciente, com média de 10,48 atendimentos por paciente no período de acompanhamento da coorte, com mediana de cerca de seis meses. Os resultados mostraram ainda valor médio de custo por atendimento por paciente de $\mathrm{R} \$$ 11.282,19. Quando os cenários foram analisados, esse custo variou de R\$ 6.162 em ambiente ambulatorial a $\mathrm{R} \$ 40.468$ em ambiente hospitalar. Porém, quanto à quantidade de atendimentos, os ambulatoriais foram mais frequentes, o que pode ser justificado pelo acompanhamento mais próximo e pela necessidade de visitas ambulatoriais recorrentes para o tratamento de câncer de próstata com metástase óssea e suas complicações.

A maioria dos pacientes recebeu mais de um tipo de tratamento durante o período de acompanhamento e os custos com medicamentos representaram o maior impacto econômico entre todos os custos médicos relacionados aos atendimentos da coorte.

A avaliação apresentada é apenas analítica, não permitindo inferências sobre uma possível comparação entre coortes com ou sem metástase óssea. Um estudo brasileiro avaliou que os custos médicos por paciente-ano relacionados ao câncer de próstata em estágios iniciais para uma organização do Sistema de Saúde Suplementar são de R\$ 432 para tratamento expectante, $\mathrm{R} \$ 4.641$ para pacientes em terapia localizada, $\mathrm{R} \$$ 5.850 para aqueles em TH e R\$33.773 para o grupo em uso de quimioterapia (QT) (Santos et al., 2014). Tais valores contrastam de forma relevante com o encontrado nesta análise, que chegou a R\$ 118.183 por paciente durante uma mediana de apro-

Tabela 4. Ambiente ambulatorial por tipo de tratamento: custo por paciente, número de atendimentos por paciente, custo por atendimento

\begin{tabular}{lcccc}
\hline Desfecho & TH $(\mathbf{n}=\mathbf{7 4 )}$ & $\mathbf{R T}(\mathbf{n}=\mathbf{1 0 1})$ & TO $(\mathbf{n}=\mathbf{9 6})$ & PO $(\mathbf{n}=\mathbf{6})$ \\
\hline Custo por paciente & $\mathrm{R} \$ 17.445,29$ & $\mathrm{R} \$ 4.186,03$ & $\mathrm{R} \$ 17.110,05$ & $\mathrm{R} \$ 2.734,59$ \\
\hline Número de atendimentos & 259 & 135 & 596 & 6 \\
\hline Atendimentos por paciente & 3,50 & 1,34 & 6,42 & 1 \\
\hline Intervalo entre atendimentos (dias) & 115,28 & 204,80 & 56,90 & - \\
\hline Custo por atendimento & $\mathrm{R} \$ 4.984,37$ & $\mathrm{R} \$ 3.131,77$ & $\mathrm{R} \$ 2.755,98$ & $\mathrm{R} \$ 2.734,59$ \\
\hline
\end{tabular}

TH: terapia hormonal; RT: radioterapia; TO: terapia oncológica; PO: procedimento ósseo.

Tabela 5. Custo hospitalar por tipo de tratamento

\begin{tabular}{|c|c|c|c|c|}
\hline Tipo de custo & $\mathrm{TH}(\mathrm{n}=66)$ & RT (n = 14) & TO $(n=14)$ & $\mathrm{PO}(\mathrm{n}=35)$ \\
\hline Medicamentos & $\mathrm{R} \$ 1.505 .413,82(26,54 \%)$ & $\mathrm{R} \$ 2.914,20(3,81 \%)$ & $\mathrm{R} \$ 655.609,05(50,74 \%)$ & $\mathrm{R} \$ 66.342,59(7,68 \%)$ \\
\hline Materiais & $\mathrm{R} \$ 1.984 .667,91$ (34,99\%) & $\mathrm{R} \$ 2.112,45(2,77 \%)$ & $\mathrm{R} \$ 299.424,38(23,17 \%)$ & $\mathrm{R} \$ 491.242,38(56,90 \%)$ \\
\hline Taxas diversas & $\mathrm{R} \$ 1.157 .791,72(20,41 \%)$ & $\mathrm{R} \$ 6.903,09$ (9,04\%) & R\$ 164.992,98 (12,77\%) & R\$ 90.826,92 (10,52\%) \\
\hline Procedimentos & $\mathrm{R} \$ 444.943,50$ (7,85\%) & R\$ 59.128,35 (77,40\%) & $\mathrm{R} \$ 50.857,72(3,94 \%)$ & $\mathrm{R} \$ 165.682,07$ (19,19\%) \\
\hline Exames & $\mathrm{R} \$ 326.816,82(5,76 \%)$ & $\mathrm{R} \$ 1.037,17(1,36 \%)$ & $\mathrm{R} \$ 92.291,08(7,14 \%)$ & $\mathrm{R} \$ 35.612,36(4,12 \%)$ \\
\hline Gases medicinais & R\$ 169.466,77 (2,99\%) & - & $\mathrm{R} \$ 28.980,10(2,24 \%)$ & $\mathrm{R} \$ 10.131,70(1,17 \%)$ \\
\hline Pacotes & $\mathrm{R} \$ 82.537,03(1,46 \%)$ & $\mathrm{R} \$ 4.293,67$ (5,62\%) & - & $R \$ 3.528,17(0,41 \%)$ \\
\hline Total & $\mathrm{R} \$ 5.671 .637,56(100,00 \%)$ & $\mathrm{R} \$ 76.388,93(100,00 \%)$ & $\mathrm{R} \$ 1.292 .155,30(100,00 \%)$ & $\mathrm{R} \$ 863.366,19(100,00 \%)$ \\
\hline
\end{tabular}

HT: terapia hormonal; RT: radioterapia; TO: terapia oncológica; PO: procedimento ósseo. 
Tabela 6. Ambiente hospitalar por tipo de tratamento: custo por paciente, número de atendimentos por paciente, custo por atendimento e custo por dia de atendimento

\begin{tabular}{|c|c|c|c|c|}
\hline Desfecho & TH $(n=66)$ & $\mathrm{RT}(\mathrm{n}=14)$ & TO $(n=14)$ & $\mathrm{PO}(\mathrm{n}=35)$ \\
\hline Custo por paciente & $R \$ 85.933,90$ & $R \$ 5.456,35$ & $\mathrm{R} \$ 92.296,81$ & $R \$ 24.667,61$ \\
\hline Número de atendimentos & 122 & 17 & 22 & 38 \\
\hline Atendimentos por paciente & 1,85 & 1,21 & 1,57 & 1,09 \\
\hline Intervalo entre atendimentos (dias) & 125,77 & 89,57 & 212,20 & 121,62 \\
\hline Custo por atendimento & $R \$ 42.486,31$ & $R \$ 4.106,60$ & $R \$ 53.677,52$ & $R \$ 20.764,04$ \\
\hline Tempo médio por atendimento (dias) & 10,71 & 1,06 & 11,86 & 4,13 \\
\hline Custo por dia de atendimento & $\mathrm{R} \$ 4.339,43$ & $R \$ 4.243,83$ & $R \$ 4.950,79$ & $R \$ 5.499,15$ \\
\hline
\end{tabular}

TH: terapia hormonal; RT: radioterapia; TO: terapia oncológica; PO: procedimento ósseo.

ximadamente seis meses de acompanhamento. O evidente contraste supostamente se dá pelo fato de esta análise incluir pacientes com metástases ósseas, o que demonstra que tais pacientes demandam maiores custos de tratamento em comparação aos em estágios iniciais da doença.

Por ser uma análise baseada em um banco de dados de uma empresa privada, a principal limitação deste estudo está relacionada à qualidade dos dados relatados no banco e ao tamanho da amostra, o que pode potencialmente levar à interpretação de dados com viés. É importante enfatizar que existem regras específicas no SSS do Brasil que não obrigam os provedores a reportarem alguns campos nas faturas médicas, como o registro por CID, gênero e faixa etária dos pacientes, gerando perda de parte dessas informações. Embora haja uma amostra inicial considerável, houve uma perda substancial de acompanhamento de pacientes com doença avançada no caminho da análise, em grande parte por falta de registro por CID, especialmente aquele voltado à metástase óssea. O estudo não apresenta potencial para generalizações amplas e não foi realizado cálculo de tamanho amostral, uma vez que se trata de avaliação de banco com dados no cenário de mundo real. Além disso, outra limitação a ser mencionada é o curto horizonte temporal adotado na análise, que neste estudo foi a partir de janeiro de 2010. Além disso, há falta de detalhes nos tratamentos disponíveis em razão da ampla terminologia nos registros da tabela nacional de Saúde Suplementar.

Apesar das limitações apontadas, as evidências obtidas de estudos de mundo real são de extrema importância para o aumento da conscientização sobre o atual gerenciamento de doenças e para tornar conhecido e tangível aos pagadores o seu impacto econômico. Pacientes com metástases ósseas geram mais custos, pois a elevada necessidade de hospitalizações e maior tempo médio de permanência resultam em gastos superiores aos demandados por pacientes sem metástases ósseas. A prevenção e o tratamento desses pacientes são críticos para uma gestão de custos eficiente.
Este foi o primeiro estudo a descrever a utilização de recursos e os custos médicos hospitalares associados ao tratamento do câncer de próstata com metástase óssea, na perspectiva do SSS brasileiro.

\section{Conclusão}

O presente estudo corrobora o alto custo das intervenções aplicadas a pacientes com câncer de próstata com metástase óssea. Apesar das limitações relacionadas à amostragem e à qualidade dos dados fornecidos, o estudo demonstrou que o impacto econômico para o SSS brasileiro superou, aproximadamente três vezes, o impacto com o tratamento de pacientes em estágios iniciais da doença. Dessa forma, o tratamento do câncer de próstata com metástase óssea apresenta elevada carga econômica no contexto do SSS brasileiro, portanto a atenuação dos sintomas resultantes das metástases ósseas é essencial para reduzir as hospitalizações e, consequentemente, essa carga.

\section{Agradecimentos}

Agradecemos a participação de Flavia Toburella pelas contribuições realizadas no desenvolvimento desta análise.

\section{Referências bibliográficas}

Agência Brasil. Empresa Brasil de Comunicação. Centro de Diagnóstico do Câncer de Próstata [Internet]. 2017 [cited 2018 Jan 29]. Disponível em: http://agenciabrasil.ebc.com.br/geral/noticia/2017-11/centro-dediagnostico-do-cancer-de-prostata-fara-36-mil-biopsias-por-ano. Acesso em: 14 ago 2018.

Bubendorf L, Schöpfer A, Wagner U, Sauter G, Moch H, Willi N, et al. Metastatic patterns of prostate cancer: an autopsy study of 1,589 patients. Hum Pathol. 2000 May;31(5):578-83.

Chi KN, Bjartell A, Dearnaley D, Saad F, Schröder FH, Sternberg C, et al. Castration-resistant prostate cancer: from new pathophysiology to new treatment targets. Eur Urol. 2009 Oct;56(4):594-605. 
Gandaglia G, Abdollah F, Schiffmann J, Trudeau V, Shariat SF, Kim SP, et al. Distribution of metastatic sites in patients with prostate cancer: a population-based analysis. Prostate. 2014 Feb;74(2):210-6.

Instituto Brasileiro de Geografia e Estatística (IBGE). Sistema nacional de índices de preços ao consumidor IPCA e INPC [Internet]. 2017 Dez. Disponível em: https://biblioteca.ibge.gov.br/visualizacao/ periodicos/236/inpc_ipca_2017_dez.pdf. Acesso em: 14 ago 2018.

Jerez-Roig J, Souza DLB, Medeiros PFM, Barbosa IR, Curado MP, Costa ICC, et al. Future burden of prostate cancer mortality in Brazil: a populationbased study. Cad Saúde Pública. 2014;30(11):2451-8.

Manca P, Pantano F, luliani M, Ribelli G, De Lisi D, Danesi R, et al. Determinants of bone specific metastasis in prostate cancer. Crit Rev Oncol Hematol. 2017:112:59-66.

Ministério da Saúde (Brasil). Instituto Nacional de Câncer José Alencar Gomes da Silva (INCA). Estimativa 2018: incidência de câncer no Brasil. Rio de Janeiro: Ministério da Saúde, 2018.

Ministérios da Saúde (Brasil). Instituto Nacional de Câncer José Alencar Gomes da Silva (INCA). Câncer de prostáta _ Sintomas [Internet]. 2016a. Disponível em: http://www2.inca.gov.br/wps/wcm/connect/ tiposdecancer/site/home/prostata/sintomas. Acesso em: 14 ago 2018.
Ministério da Saúde (Brasil). Conselho Nacional de Saúde. Resolução n 510, de 7 de abril de 2016 [Internet]. 2016b. Disponível em: http://conselho. saude.gov.br/resolucoes/2016/Res0510.pdf. Acesso em: 14 ago 2018.

National Cancer Institute. Cancer of the Prostate-SEER Stat Fact Sheet.

Santos M, Luiz C, Maturana M. Direct medical costs (DMC) of treating prostate cancer in a medical cooperative HMO in Brazil: results from a longitudinal analysis of an administrative database. Value Health. 2014;17(3):A80.

Seal B, Sullivan SD, Ramsey SD, Asche CV, Shermock K, Sarma S, et al. Comparing hospital-based resource utilization and costs for prostate cancer patients with and without bone metastases. Appl Health Econ Health Policy. 2014;12(5):547-57.

Shore ND. Radium-223 dichloride for metastatic castration-resistant prostate cancer: the urologist's perspective. Urology. 2015 Apr;85(4):717-24.

World Health Organization (WHO). GLOBOCAN 2012: estimated cancer incidence, mortality and prevalence wordwide in 2012. 